Supporting Information

\title{
$n$-Type $\mathrm{Bi}_{2} \mathrm{Te}_{3-\mathrm{x}} \mathrm{Se}_{\mathrm{x}}$ Nanoplates with Enhanced
}

\section{Thermoelectric Efficiency Driven by Wide-}

\section{Frequency Phonon Scatterings and Synergistic}

\section{Carrier Scatterings}

Min Hong, ${ }^{\dagger}$ Thomas C. Chasapis, ${ }^{\ddagger}{ }^{\S}$ Zhi-Gang Chen, ${ }^{*} \dagger$ Lei Yang, ${ }^{\dagger}$ Mercouri G. Kanatzidis, ${ }^{\S}$ G. Jeffrey Snyder, ${ }^{*}+\underset{+}{*}$ and Jin Zou ${ }^{*}, \dagger, \|$

${ }^{\dagger}$ Materials Engineering, University of Queensland, Brisbane, QLD 4072, Australia

Department of Materials Science and Engineering, Northwestern University, Evanston, Illinois 60208, USA

${ }^{\S}$ Department of Chemistry, Northwestern University, Evanston, Illinois 60208, USA

${ }^{\|}$Centre for Microscopy and Microanalysis, University of Queensland, Brisbane, QLD 4072, Australia

${ }^{*}$ Address correspondence to

E-mail: j.zou@uq.edu.au, z.chen1@uq.edu.au,and jeff.snyder@,northwestern.edu

KEYWORDS: $\mathrm{Bi}_{2} \mathrm{Te}_{3-\mathrm{x}} \mathrm{Se}_{\mathrm{x}}$, nanostructuring, thermoelectric, synergistic carrier scatterings, wide-frequency phonon scatterings 


\section{Determination of Lattice Parameters}

It is well known that the relationship between the lattice spacing of the atomic planes $\left(d_{h k i l}\right)$ and its lattice parameters ( $a$ and $c$ ) for rhombohedral structure can be calculated by ${ }^{1}$

$$
\frac{1}{d_{h k l}^{2}}=\frac{4}{3}\left(\frac{h^{2}+h k+k^{2}}{a^{2}}\right)+\frac{l^{2}}{c^{2}},
$$

where hkil are the index of the $\{h k i l\}$ atomic planes. Since $d_{h k i l}$ can be measured from XRD patterns, different $a$ and $c$ can be determined for different $\mathrm{Bi}_{2} \mathrm{Te}_{3-\mathrm{x}} \mathrm{Se}_{\mathrm{x}}$ samples. By using the changes of $00015^{*}$ and $01 \overline{1} 5^{*}$ in their $2 \theta$, the corresponding $c$ and $a$ can be determined. Figure S1 shows the determined $a$ and $c$ as a function of the Se content, in which the measured variation of lattice parameters follow the Vegard's law.

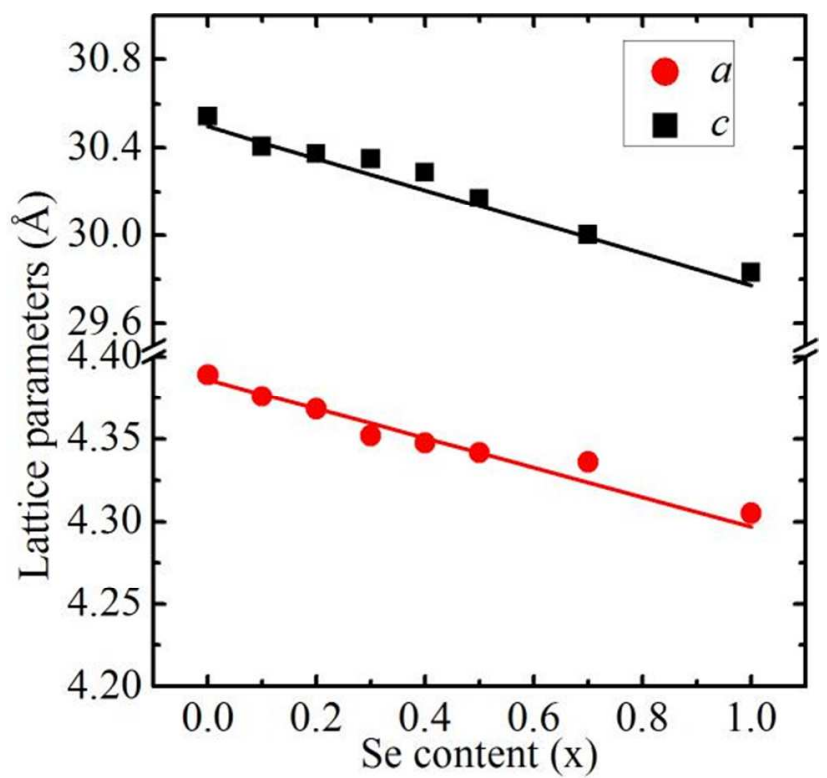

Figure S1 Determined lattice parameters as a function of the Se content for different as-prepared $\mathrm{Bi}_{2} \mathrm{Te}_{3-\mathrm{x}} \mathrm{Se}_{\mathrm{x}}$ nanoplates. 


\section{Chemical Composition Determination Using EDS}

The chemical compositions of different as-synthesized $\mathrm{Bi}_{2} \mathrm{Te}_{3-\mathrm{x}} \mathrm{Se}_{\mathrm{x}}$ nanoplates were determined by EDS (equipped in TEM). Figure S2 shows the EDS profiles corresponding to different $\mathrm{x}$, in which the variations of the $\mathrm{Bi}, \mathrm{Te}$, and Se peaks can be seen for samples with $0<\mathrm{x} \leq 1$ (note that the $\mathrm{Cu}$ peaks are originated from the $\mathrm{Cu}$ TEM grids). Detailed quantitative analysis indicates that elemental ratios of $\mathrm{Bi}, \mathrm{Te}$, and $\mathrm{Se}$ are close to the nominal compositions.

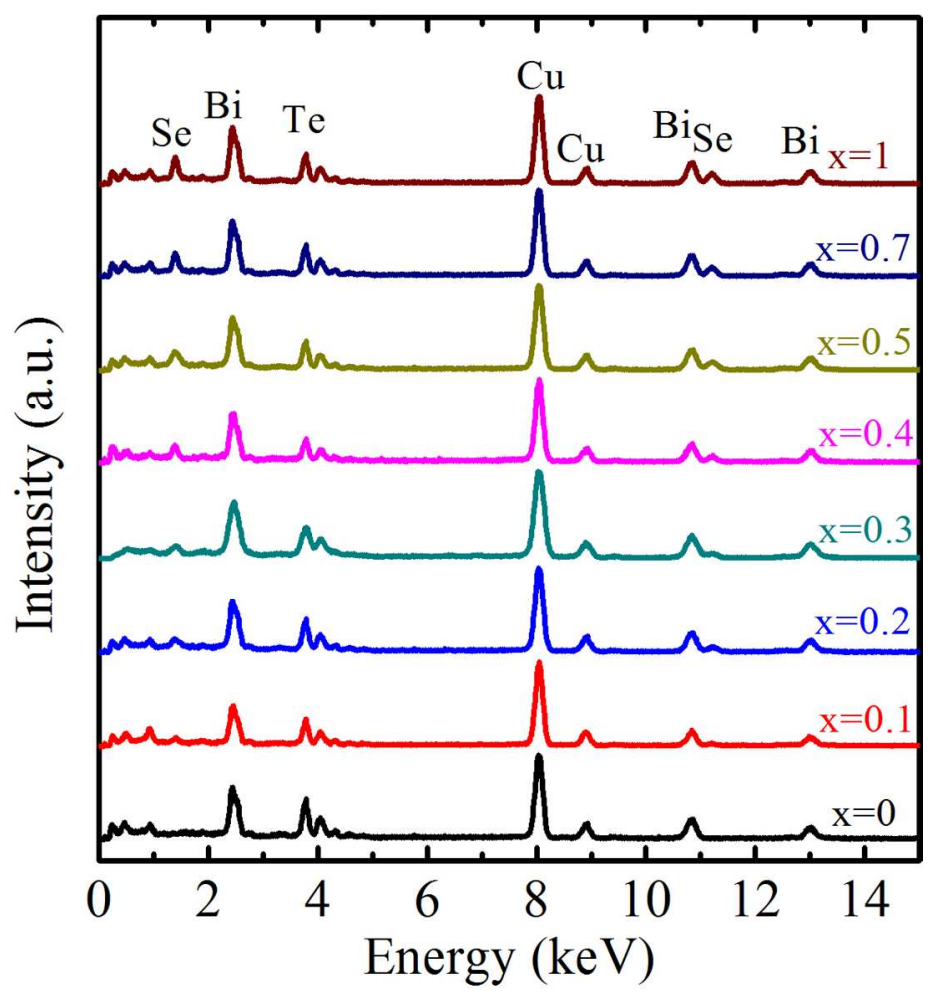

Figure S2 EDS profiles of as-synthesized $\mathrm{Bi}_{2} \mathrm{Te}_{3-\mathrm{x}} \mathrm{Se}_{\mathrm{x}}$ nanostructures. 


\section{SEM Characterization of $\mathrm{Bi}_{2} \mathrm{Te}_{2.7} \mathrm{Se}_{0.3}$ Nanostructures Prepared with and without PVP}

Figure $\mathrm{S} 3 \mathrm{a}$ and $\mathrm{b}$ are $\mathrm{SEM}$ images of $\mathrm{Bi}_{2} \mathrm{Te}_{2.7} \mathrm{Se}_{0.3}$ nanoplates synthesized without surfactant and with poly(N-vinyl-2-pyrrolidone) (PVP, $\left.M_{w}=40,000\right)$ serving as the surfactant, respectively. As can be seen, the $\mathrm{Bi}_{2} \mathrm{Te}_{2.7} \mathrm{Se}_{0.3}$ nanoplates synthesized with PVP is more uniform, and their edges and corners are clearer, compared with the nanoplates synthesized without PVP. The thicknesses of the two kinds of nanoplates are nearly identical $(\sim 30 \mathrm{~nm})$.
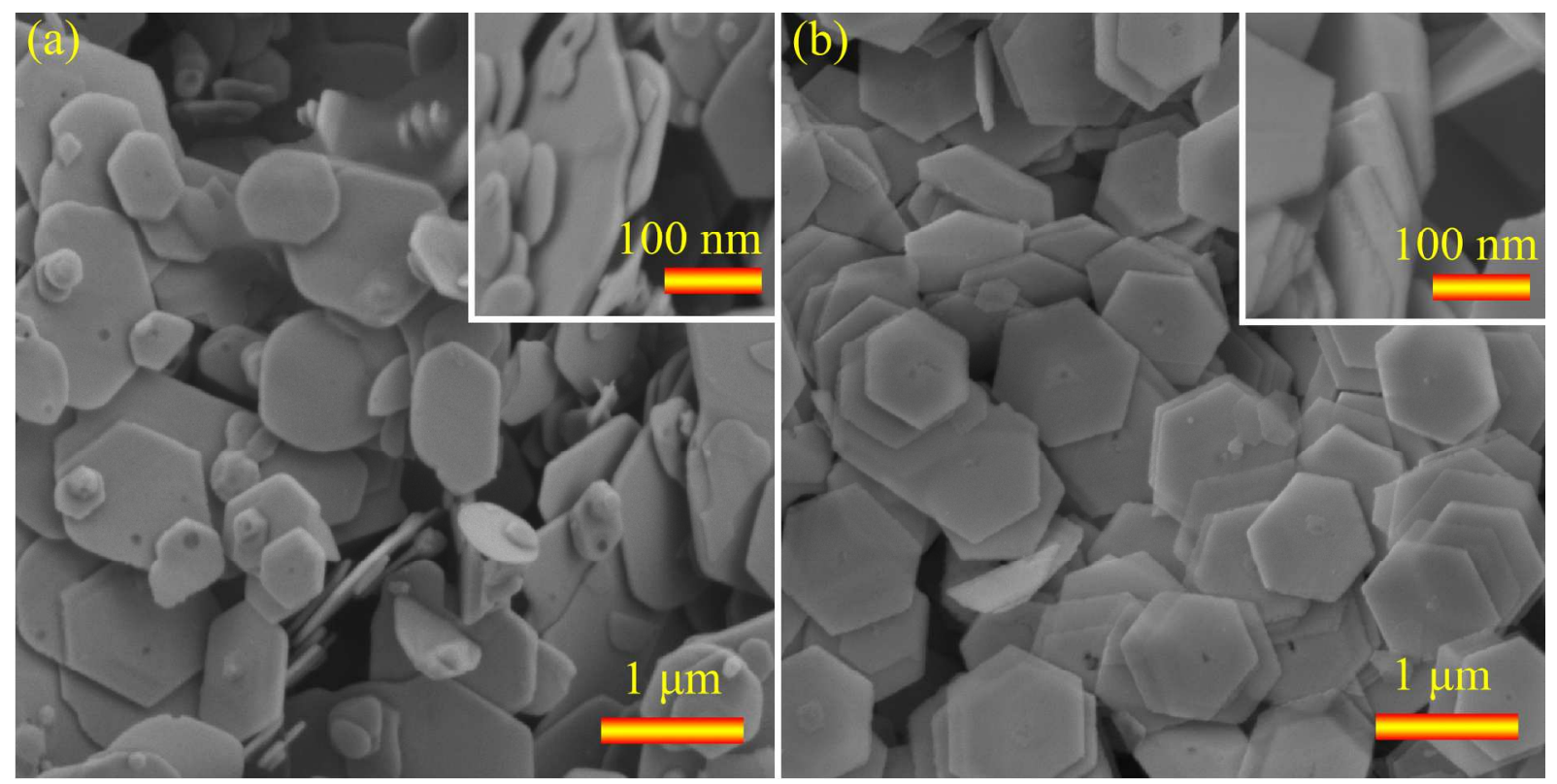

Figure S3 SEM images of $\mathrm{Bi}_{2} \mathrm{Te}_{2.7} \mathrm{Se}_{0.3}$ nanoplates prepared (a) without and (b) with PVP. The insets show the thicknesses of the nanoplates 


\section{Size Distribution of as-Synthesized $\mathrm{Bi}_{2} \mathrm{Te}_{3-\mathrm{x}} \mathrm{Se}_{\mathrm{x}}$ Nanoplates}

To examine the size distribution of as-synthesized $\mathrm{Bi}_{2} \mathrm{Te}_{3-\mathrm{x}} \mathrm{Se}_{\mathrm{x}}$ nanoplates, SEM characterization was employed. Figure S4 summarizes SEM images of as-synthesized nanoplates. Based on this, lateral size distributions were obtained and shown in Figure S5, from which the majority size of nanoplates for all compositions is in the range of $\sim 1 \mu \mathrm{m}$.

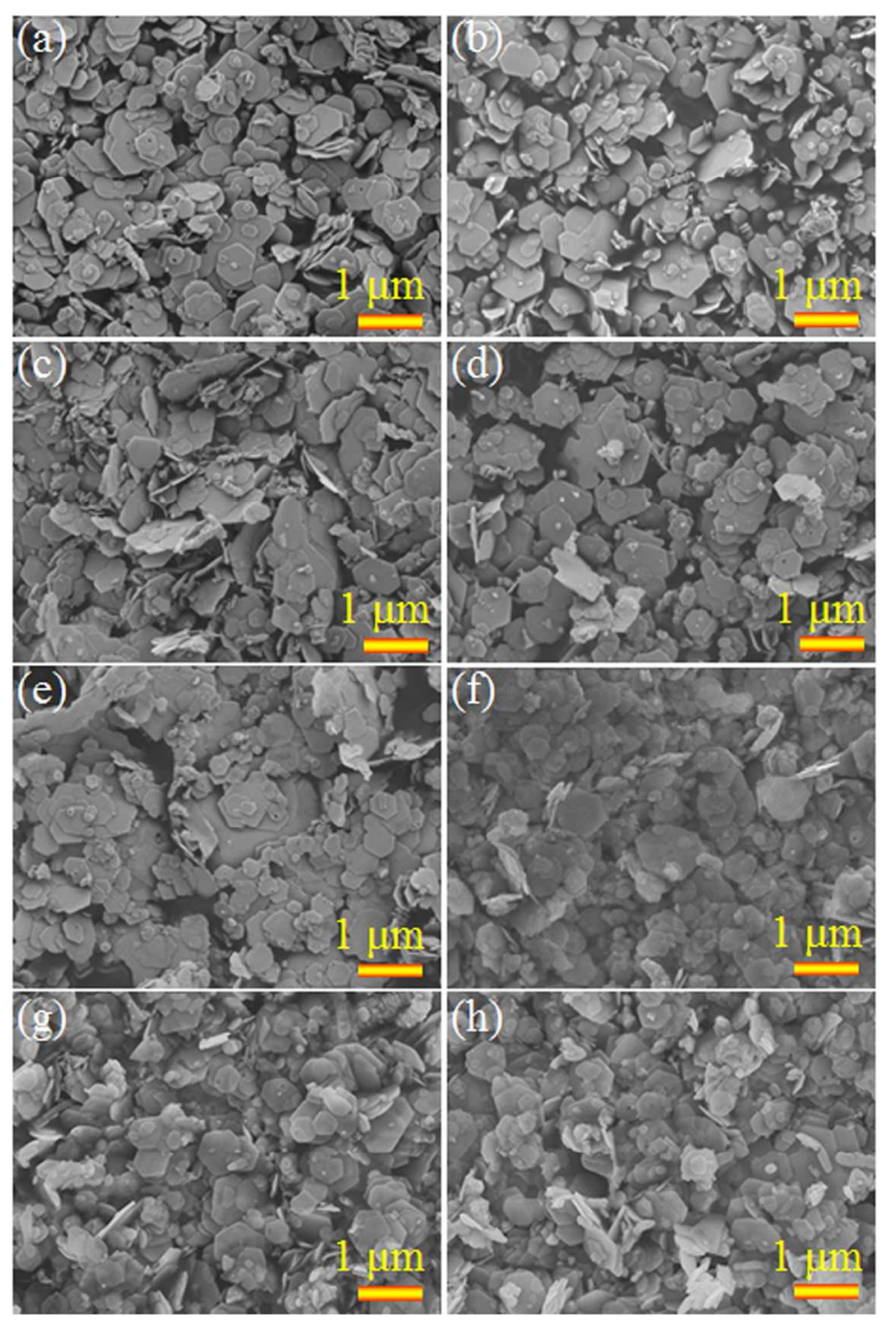

Figure S4 SEM images of $\mathrm{Bi}_{2} \mathrm{Te}_{3-\mathrm{x}} \mathrm{Se}_{\mathrm{x}}$ nanoplates: (a) $\mathrm{x}=0$, (b) $\mathrm{x}=0.1$, (c) $\mathrm{x}=0.2$, (d) $\mathrm{x}=0.3$, (e) $\mathrm{x}=0.4,(\mathrm{f}) \mathrm{x}=0.5,(\mathrm{~g}) \mathrm{x}=0.7$, and (h) $\mathrm{x}=1$. 

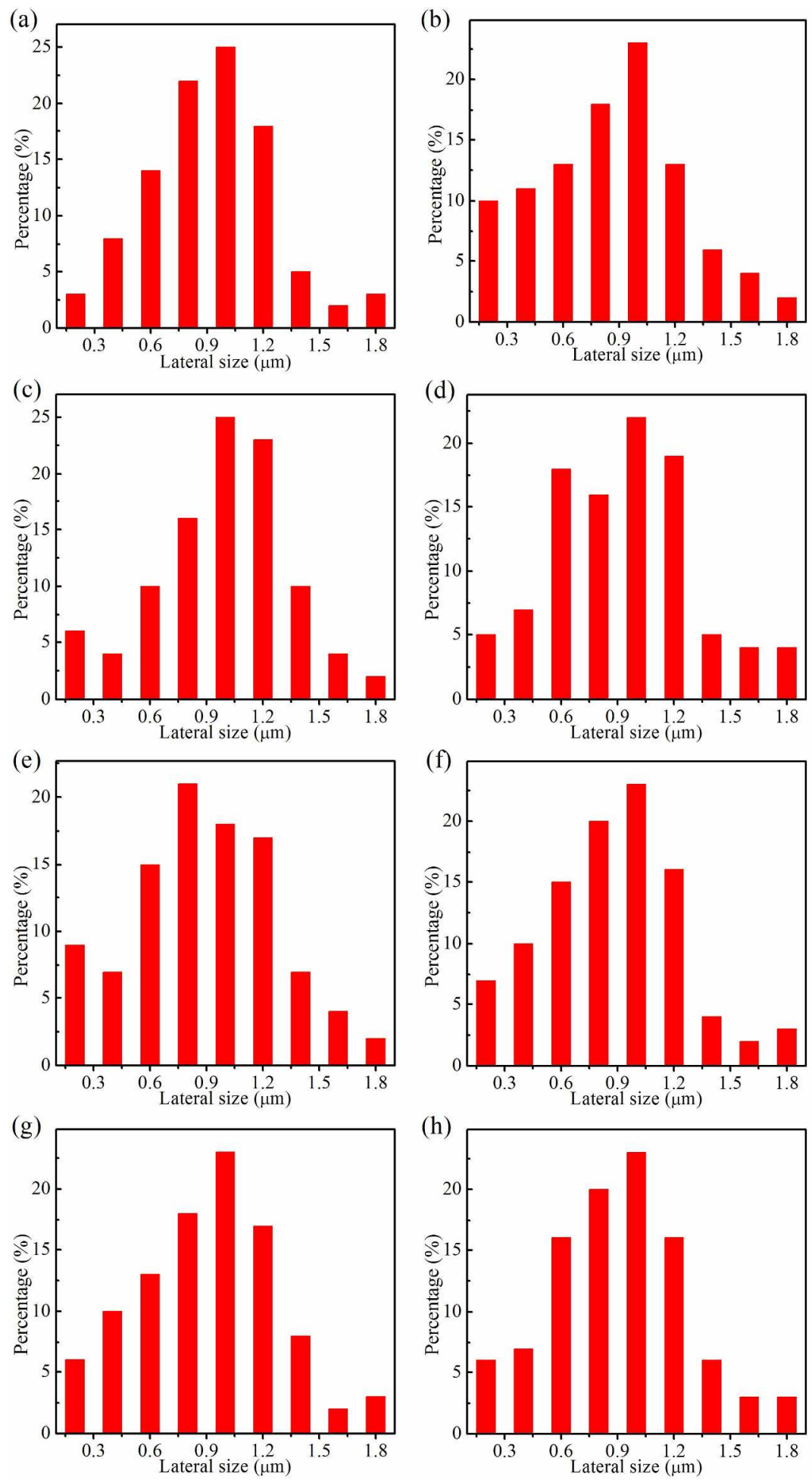

Figure S5 Statistical lateral size distributions of $\mathrm{Bi}_{2} \mathrm{Te}_{3-\mathrm{x}} \mathrm{Se}_{\mathrm{x}}$ nanoplates: (a) $\mathrm{x}=0$, (b) $\mathrm{x}=0.1$, (c) $\mathrm{x}=0.2,(\mathrm{~d}) \mathrm{x}=0.3,(\mathrm{e}) \mathrm{x}=0.4,(\mathrm{f}) \mathrm{x}=0.5,(\mathrm{~g}) \mathrm{x}=0.7$, and (h) $\mathrm{x}=1$. 


\section{XRD Patters of the Sintered Pellets and the Determination of Texture Fraction}

Spark plasma sintering was used to prepare pellets for measuring their thermoelectric properties.

Figure S6 shows XRD patterns taken from the pellets sintered from $\mathrm{Bi}_{2} \mathrm{Te}_{3-\mathrm{x}} \mathrm{Se}_{\mathrm{x}}$ nanoplates with different Se contents. As can be seen, there is no impurity in all sintered samples.

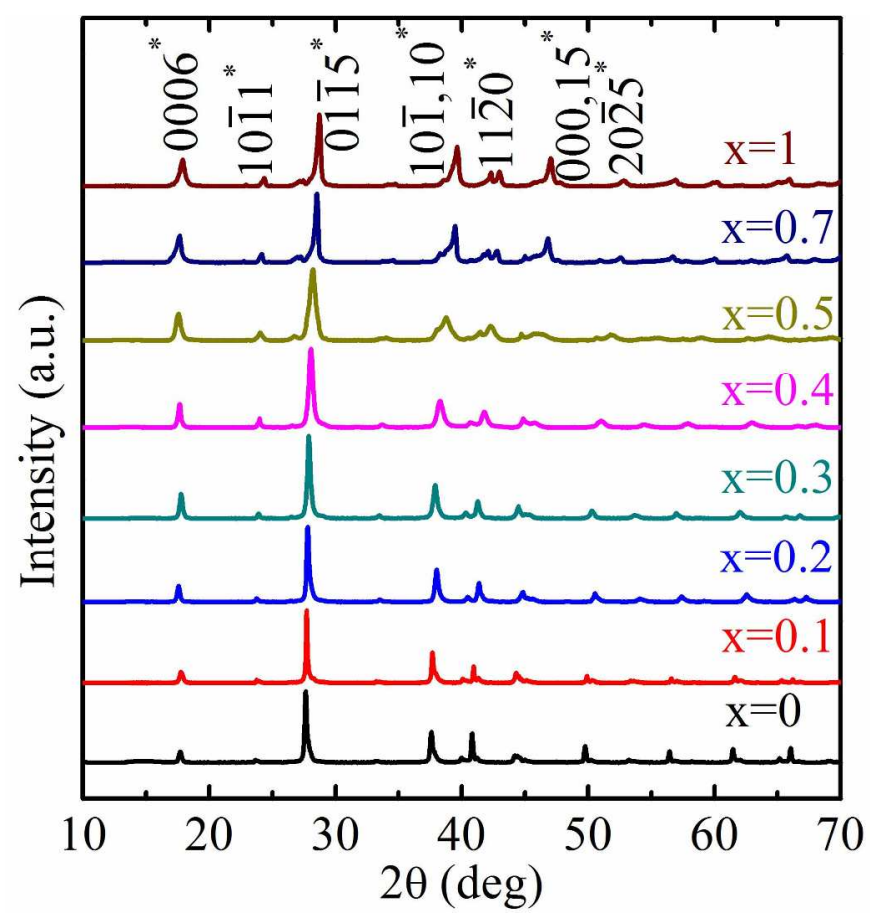

Figure S6 XRD patterns taken from pellets sintered from different $\mathrm{Bi}_{2} \mathrm{Te}_{3-\mathrm{x}} \mathrm{Se}_{\mathrm{x}}$ nanostructures.

In fact, from XRD patterns of these pellets, their texture fraction $\left(f_{h k i l}\right)$ can be determined. In this study, $f_{000 l}$ (using the $\{000 l\}$ planes) is estimated by the Lotgering method: ${ }^{2}$

$$
f_{000 l}=\frac{P_{000 l}-P_{0}}{1-P_{0}}
$$

with 


$$
P_{000 l}=\frac{\sum I_{000 l}}{\sum I_{h k i l}},
$$

and

$$
P_{0}=\frac{\sum I_{000 l}^{0}}{\sum I_{h k i l}^{0}},
$$

where $I_{h k i l}$ and $I_{h k i l}^{0}$ are the intensities of $h k i l^{*}$ diffraction peaks for our pellets and the standard values obtained from the PDF card (JCPDS No. 89-2009). Table S1 summarizes the determined texture fraction.

Table S1 Texture fractions determined using the $000 l^{*}$ reflections for $\mathrm{Bi}_{2} \mathrm{Te}_{3-\mathrm{x}} \mathrm{Se}_{\mathrm{x}}$ nanoplates and corresponding pellets.

\begin{tabular}{lllllllll}
\hline $\mathrm{x}$ & 0 & 0.1 & 0.2 & 0.3 & 0.4 & 0.5 & 0.7 & 1 \\
& & & & & & & & \\
\hline Nanoplates & 0.04 & 0.05 & 0.03 & 0.06 & 0.05 & 0.08 & 0.11 & 0.10 \\
& & & & & & & & \\
Pellets & 0.03 & 0.06 & 0.07 & 0.04 & 0.08 & 0.07 & 0.11 & 0.11 \\
& & & & & & & & \\
\hline
\end{tabular}




\section{Thermoelectric performance evaluation}

To clarify the anisotropic behavior of the pellet sintered from $\mathrm{Bi}_{2} \mathrm{Te}_{2.7} \mathrm{Se}_{0.3}$ nanoplates, thermoelectric properties were measured along in-plane and out-of-plane directions, respectively. Figure $\mathrm{S} 7$ shows the measurement results, in which $S, \sigma$ and $\kappa$ are almost identical along the two directions, indicating an isotropic nature of thermoelectric properties in our pellets, which agrees with the obtained small $f_{000 l}$ (refer to Table $\mathrm{S} 1$ ). Since $f_{000 l}$ for $\mathrm{Bi}_{2} \mathrm{Te}_{2} \mathrm{Se}$ is the largest, we also measured the thermoelectric properties for this composition along two directions, as shown in Figure S8. As can be seen, the anisotropy for $\mathrm{Bi}_{2} \mathrm{Te}_{2} \mathrm{Se}$ is slightly higher than that for $\mathrm{Bi}_{2} \mathrm{Te}_{2.7} \mathrm{Se}_{0.3}$. 

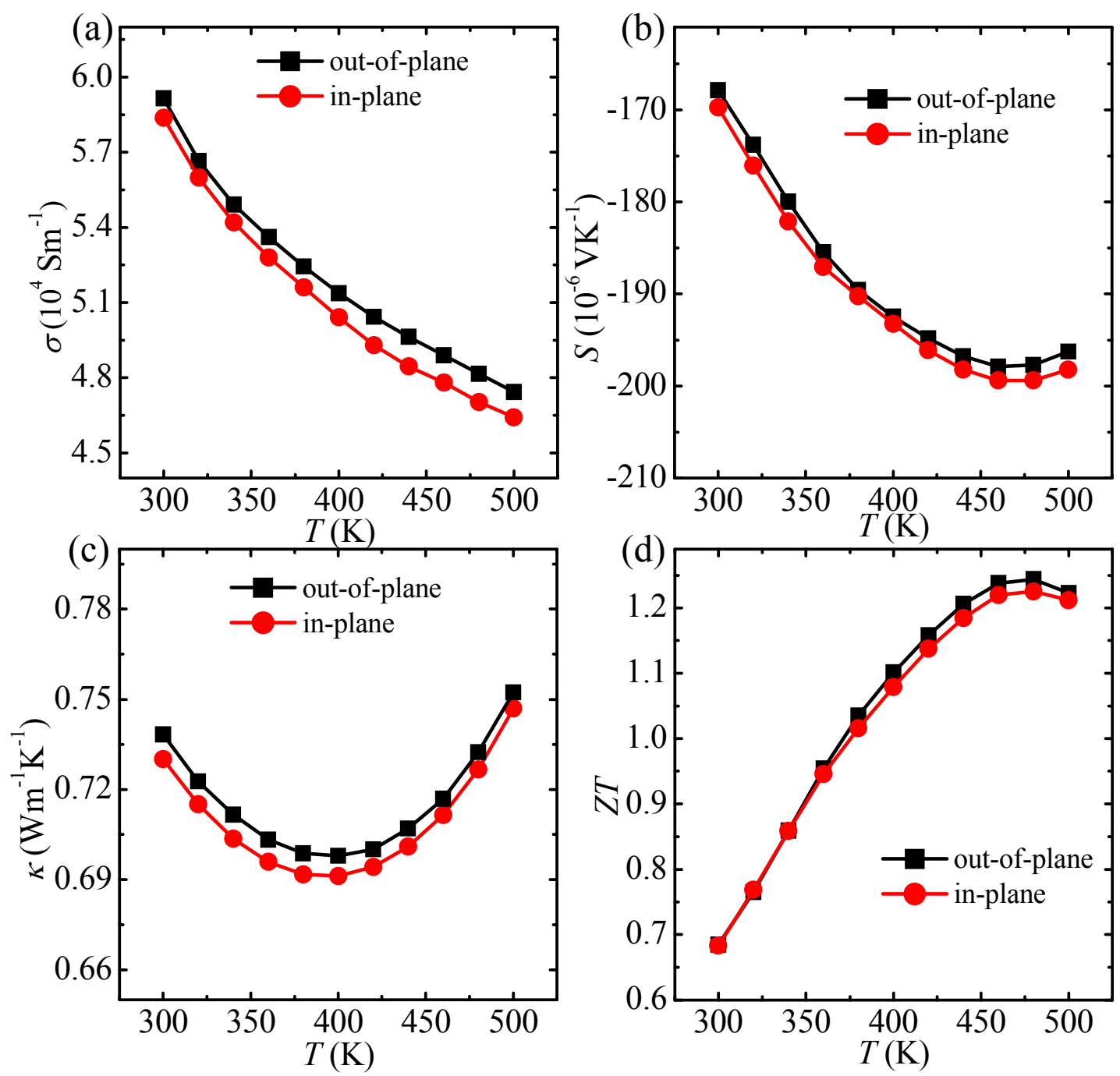

Figure S7 In-plane and out-of-plane thermoelectric characteristics of the sintered $\mathrm{Bi}_{2} \mathrm{Te}_{2.7} \mathrm{Se}_{0.3}$ pellet: (a) $\sigma$, (b) $S$, (c) $\kappa$, and (d) $Z T$; all as a function of temperature. 
(a)
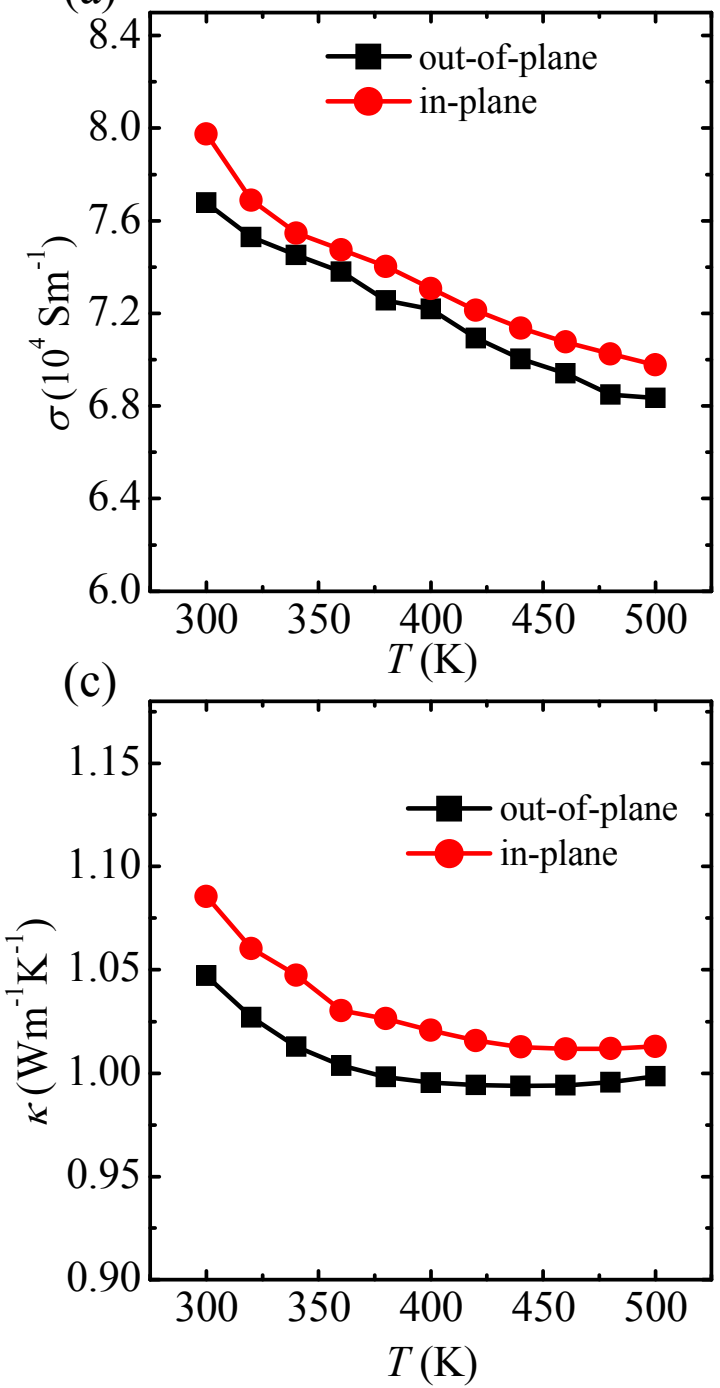

(b)

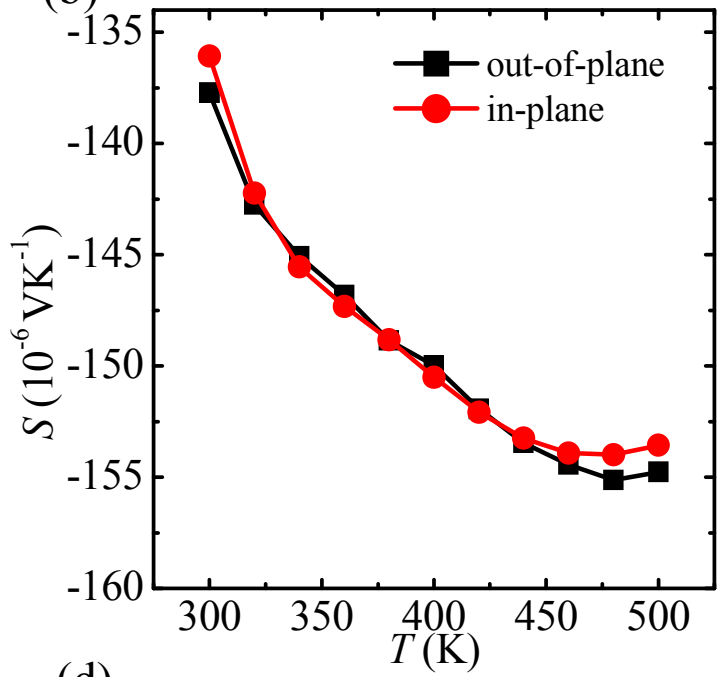

(d)

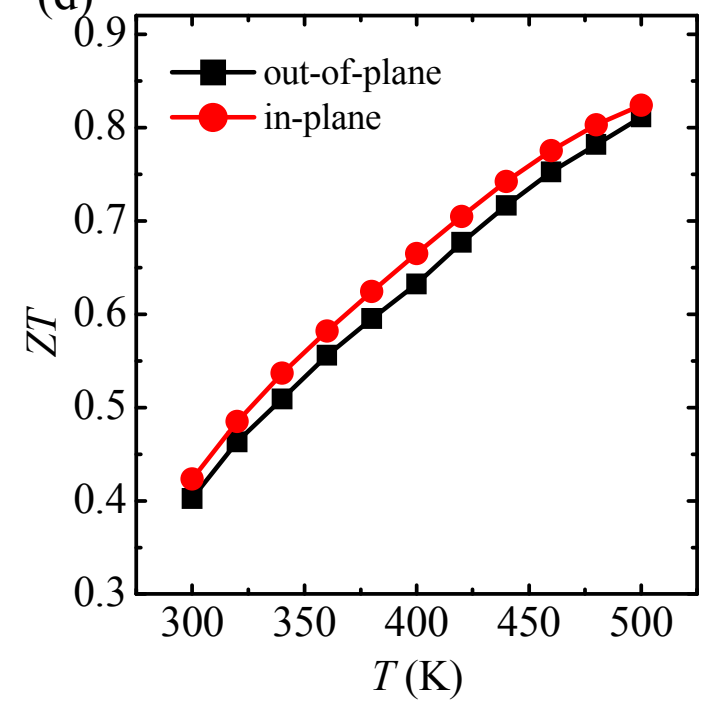

Figure S8 In-plane and out-of-plane thermoelectric characteristics of the sintered $\mathrm{Bi}_{2} \mathrm{Te}_{2} \mathrm{Se}$ pellet: (a) $\sigma$, (b) $S$, (c) $\kappa$, and (d) $Z T$; all as a function of temperature. 


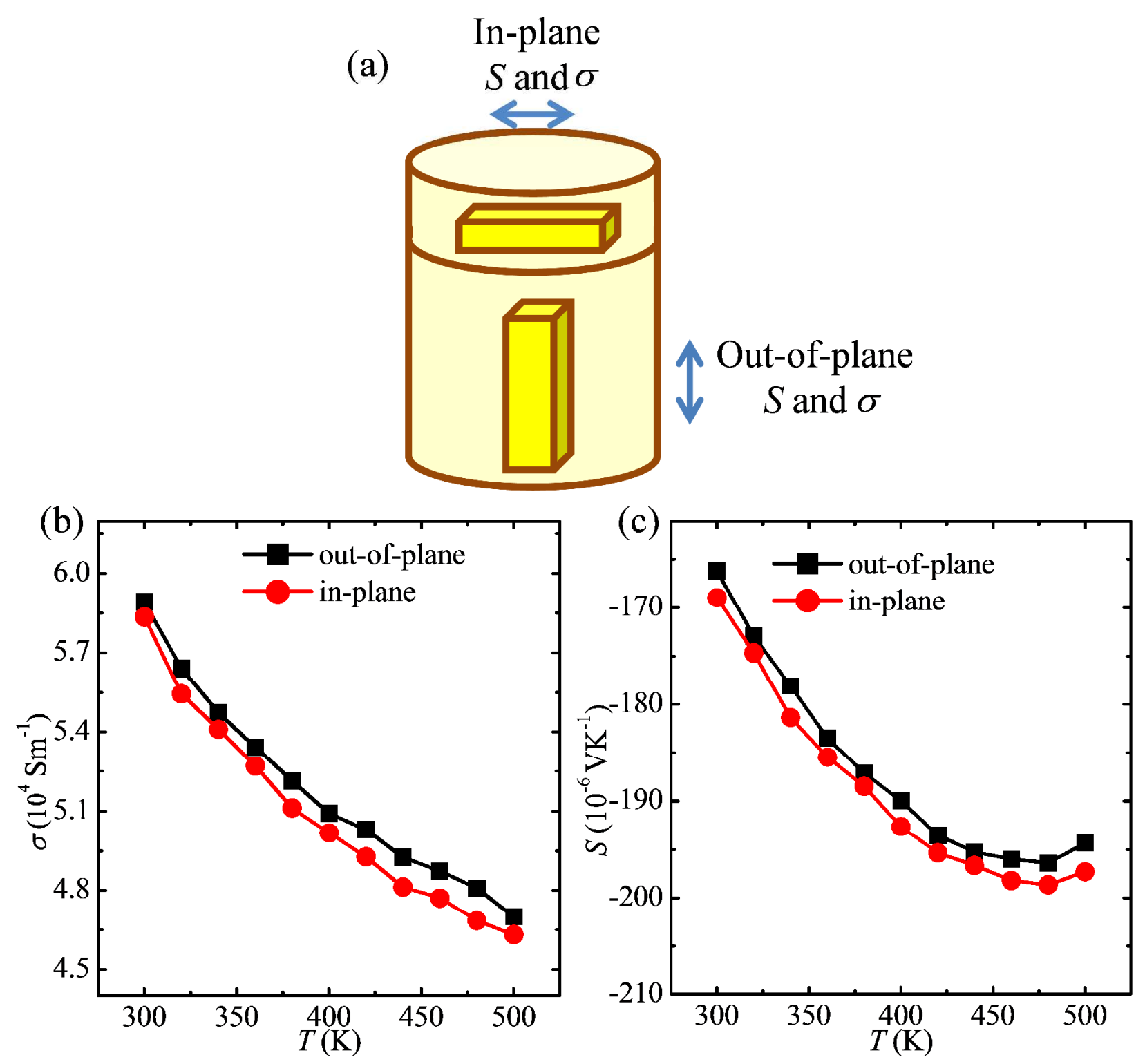

Figure S9 (a) Schematic illustrating the processing method of sintered pellet for measuring the inplane and the out-of-plane $S$ and $\sigma$. (b) and (c) The measured in-plane and out-of-plane $S$ and $\sigma$, respectively. 

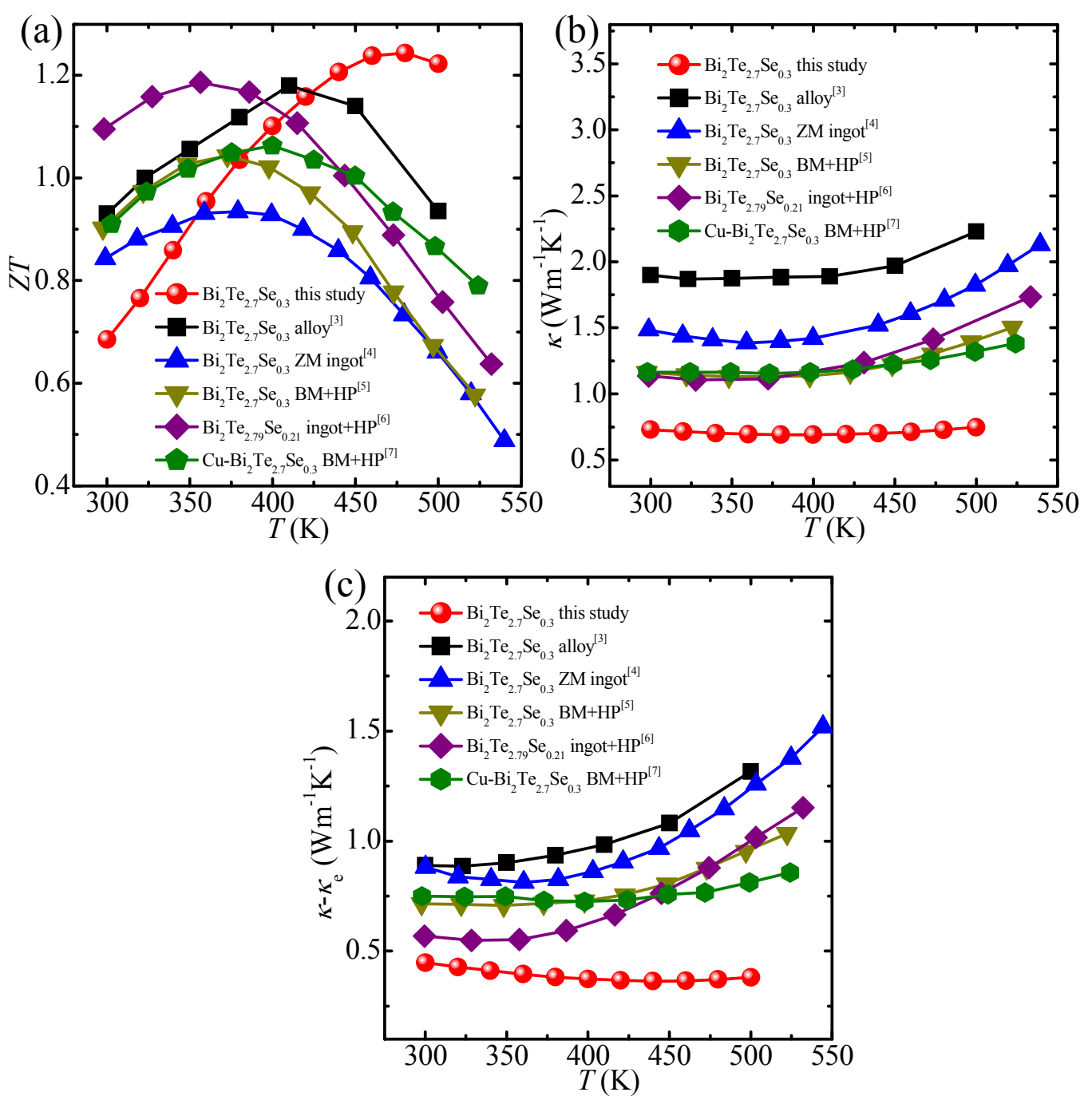

Figure S10 The temperature dependent (a) ZT, (b) $\kappa$, and (c) $\kappa-\kappa_{e}$ of $n$-type $\mathrm{Bi}_{2} \mathrm{Te}_{2.7} \mathrm{Se}_{0.3}$ for this study compared with $\mathrm{Bi}_{2} \mathrm{Te}_{2.7} \mathrm{Se}_{0.3}$ alloy Bridgman-Stockbarger method, ${ }^{3} \mathrm{Bi}_{2} \mathrm{Te}_{2.7} \mathrm{Se}_{0.3}$ zone melting (ZM) ingot, ${ }^{4} \mathrm{Bi}_{2} \mathrm{Te}_{2.7} \mathrm{Se}_{0.3}$ ball milling (BM) plus two-step dc-hot pressing (HP), $\mathrm{Bi}_{2} \mathrm{Te}_{2.79} \mathrm{Se}_{0.21}$ ingot with two-step hot pressing, ${ }^{6}$ and $\mathrm{Cu}-\mathrm{Bi}_{2} \mathrm{Te}_{2.7} \mathrm{Se}_{0.3}$ ball milling plus dc-hot pressing. $^{7}$ 


\section{Calculation of Electronic Thermal Conductivity}

According to the Wiedemann-Franz law, ${ }^{8} \kappa_{e}$ can be expressed as

$$
\kappa_{e}=L \sigma T
$$

where $L$ is the Lorenz number. Employing the single parabolic band model, $L$ can be determined by

$$
L=\left(\frac{k_{B}}{e}\right)^{2}\left[\frac{3 F_{2}(\eta)}{F_{0}(\eta)}-\left(\frac{2 F_{1}(\eta)}{F_{0}(\eta)}\right)^{2}\right],
$$

with

$$
F_{x}=\int_{0}^{\infty} \frac{\varepsilon^{x}}{1+\exp (\varepsilon-\eta)^{x}} d \varepsilon
$$

in which $e$ is free electron charge, $k_{B}$ is the Boltzmann constant, and $\eta$ is the reduced Fermi level. The determination of $\eta$ is based on the measured $S$, which is expressed as ${ }^{9}$

$$
S=\frac{k_{B}}{e}\left[\frac{2 F_{1}(\eta)}{F_{0}(\eta)}-\eta\right]
$$

From Equation (S8), $S$ varies only with $\eta$. Our previous study has revealed the monotonic relation between $S$ and $\eta{ }^{10}$ Therefore, based on the measured $S$, we can determine the corresponding $\eta$, shown in Figure S11a. Based on Equation (S6) with the determined $\eta, L$ can be determined, and their values are shown in Figure S11b. By substituting the determined $L$ and 
measured $\sigma$ over the entire studied temperature into Equation (S5), $\kappa_{e}$ can be calculated, which are shown in Figure S11c.
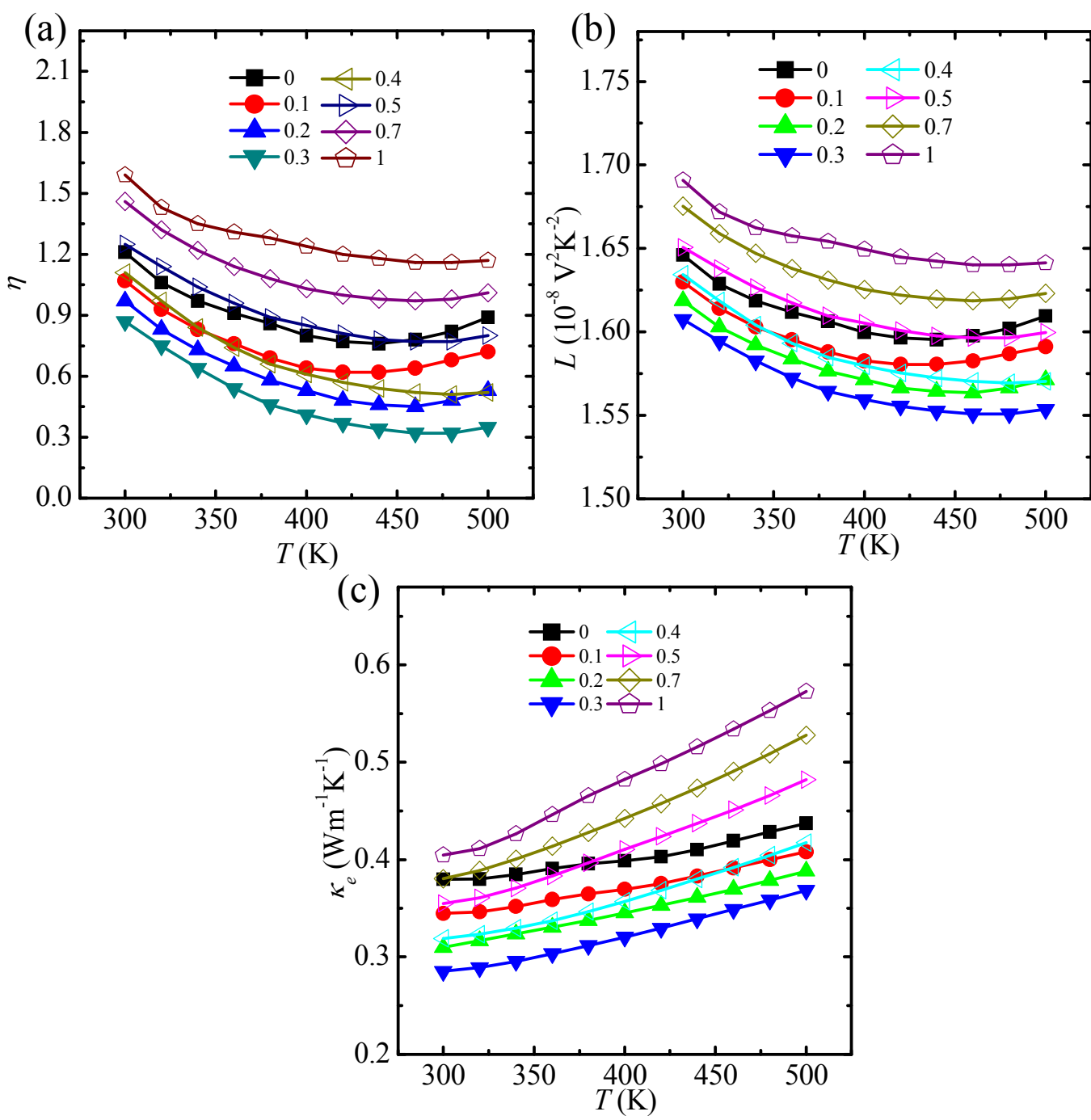

Figure S11 Composition dependent (a) $\eta$, (b) $L$, and (c) $\kappa_{e}$ as a function of temperature. 


\section{SEM Characterization of Sintered Pellets}

SEM was employed to understand the grain size distributions of sintered $\mathrm{Bi}_{2} \mathrm{Te}_{3-\mathrm{x}} \mathrm{Se}_{\mathrm{x}}$ pellets, and results are shown in Figure S12. As can be seen, the grain size is in the range of $\sim 1 \mu \mathrm{m}$, suggesting that SPS sintering did not cause notable crystal growth.
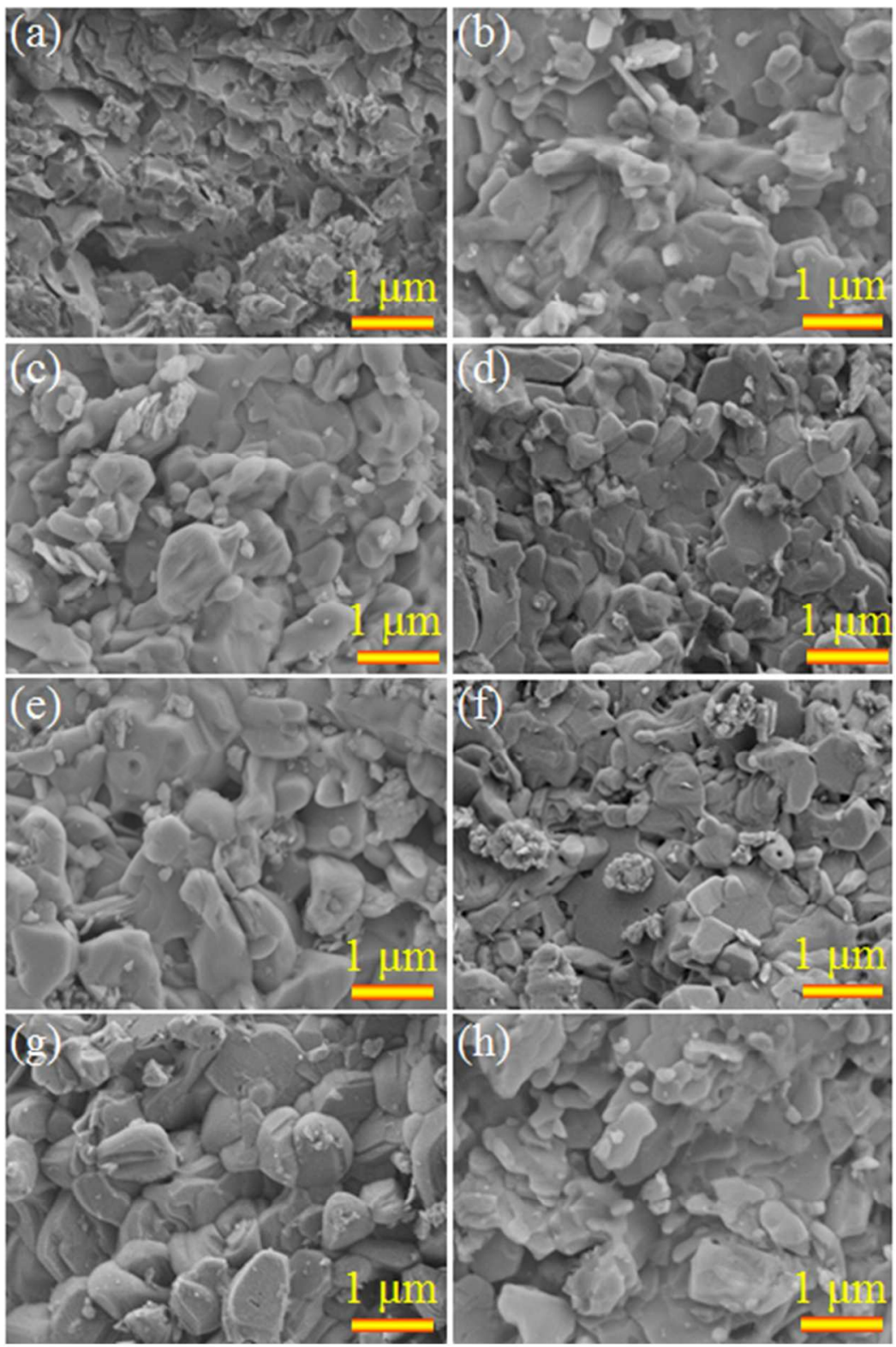

Figure S12 SEM images of sintered pellets made of $\mathrm{Bi}_{2} \mathrm{Te}_{3-\mathrm{x}} \mathrm{Se}_{\mathrm{x}}$ : (a) $\mathrm{x}=0$, (b) $\mathrm{x}=0.1$, (c) $\mathrm{x}=0.2$,

(d) $\mathrm{x}=0.3,(\mathrm{e}) \mathrm{x}=0.4$, (f) $\mathrm{x}=0.5,(\mathrm{~g}) \mathrm{x}=0.7$, and (h) $\mathrm{x}=1$. 


\section{Out-of-plane EBSD Image and the Grain Size Distribution}

To further confirm the random orietation feature, we provided another EBSD image along the out-of-plane direction, shown in Figure S13a. By taking into account both in-plane and out-ofplane EBSD images, we obtained the statistical grain size distribution of $\mathrm{Bi}_{2} \mathrm{Te}_{2.7} \mathrm{Se}_{0.3}$, shown in Figure S13b. As can be seen, the average grain size is $\sim 800 \mathrm{~nm}$.
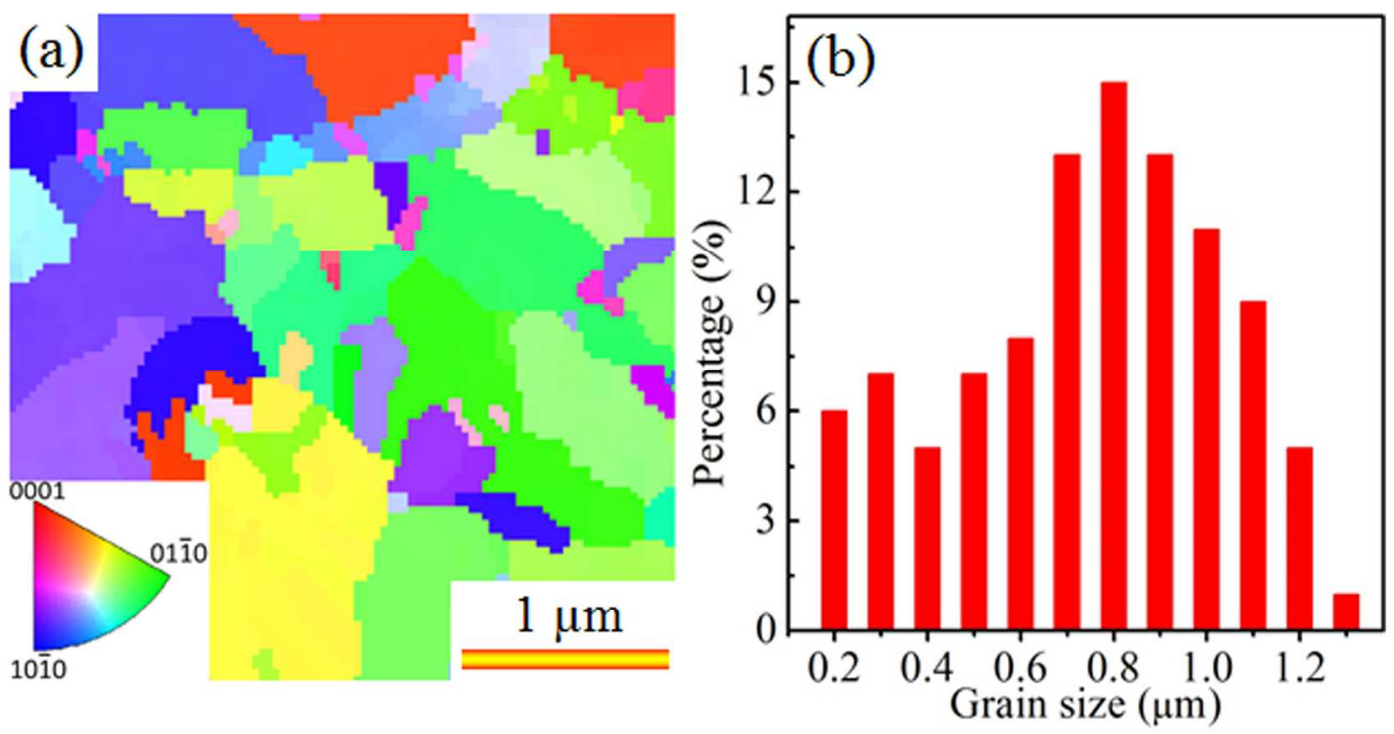

Figure $\mathrm{S} 13$ (a) EBSD image of $\mathrm{Bi}_{2} \mathrm{Te}_{2.7} \mathrm{Se}_{0.3}$ along the out-of-plane direction. (b) Statistical grain size distribution of $\mathrm{Bi}_{2} \mathrm{Te}_{2.7} \mathrm{Se}_{0.3}$ by taking into account both the in-plane (refer to Figure $5 \mathrm{a}$ of Main Manuscript) and out-of-plane EBSD images. 


\section{Calculation of Lattice Thermal Conductivity}

According to the Debye-Callaway model, ${ }^{11,12} \kappa_{l}$ can be calculated by

$$
\kappa_{l}=\frac{k_{B}}{2 \pi^{2} v}\left(\frac{k_{B} T}{\hbar}\right)^{3} \int_{0}^{\frac{\theta_{D}}{T}} \tau_{t o t} \frac{z^{4} \exp (z)}{[\exp (z)-1]^{2}} d z
$$

The integrand item in conjunction with the coefficient of Equation (S9) is the spectral lattice thermal conductivity $\left(\kappa_{s}\right),{ }^{13}$ namely

$$
\kappa_{s}=\frac{k_{B}}{2 \pi^{2} v}\left(\frac{k_{B} T}{\hbar}\right)^{3} \tau_{t o t} \frac{z^{4} \exp (z)}{[\exp (z)-1]^{2}}
$$

In the above equation, $z=\frac{\hbar \omega}{k_{B} T}$ (with $\omega$ denoting the phonon frequency) is the reduced phonon frequency, $\hbar$ is the reduced Plank constant, $\theta_{D}$ is the Debye temperature, $v=\left[\frac{1}{3}\left(\frac{1}{v_{L}^{3}}+\frac{2}{v_{T}^{3}}\right)\right]^{-1 / 3}$ (with $v_{L}$ and $v_{T}$ respectively denoting the longitudinal and transverse sound velocities) is the sound velocity, and $\tau_{\text {tot }}$ is the total relaxation time. The phonon scattering pathways generally include phonon-phonon Umklapp (U), electron-phonon (E), point defects (PD), and grain boundaries $(\mathrm{B}) .{ }^{13,14}$ The relevant phonon relaxation times are given by

Umklapp phonon scattering

$$
\tau_{U}^{-1}=\frac{\hbar \gamma^{2} \omega^{2} T}{\bar{M} v^{2} \theta_{D}} \exp \left(-\frac{\theta_{D}}{3 T}\right)
$$


Electron phonon scattering

$$
\tau_{E}^{-1}=\frac{E_{d e f}^{2} m^{*} \omega^{2}}{2 \pi \hbar^{3} \rho v_{\square}}
$$

Point defect phonon scattering

$$
\tau_{P D}^{-1}=\frac{\bar{V} \omega^{4}}{4 \pi v^{3}} \Gamma, \text { and }
$$

Grain boundary phonon scattering

$$
\tau_{B}^{-1}=\frac{v}{d}
$$

where $\gamma$ is the Grüneisen parameter, $\bar{M}$ is the average mass, $E_{\text {def }}$ is the acoustic phonon deformation potential, $m^{*}$ is the effective mass of charger carrier $\left(m^{*}=1.2 m_{0}\right.$ with $m_{0}$ representing the free electron mass), ${ }^{4} \rho$ is the sample density, $\bar{V}$ is the average atomic volume, $\Gamma$ is the point defect scattering parameter, and $d$ is the grain size, respectively.

For a material with dislocations, the scattering caused by the dislocations (D) should be considered, which includes dislocation core (DC) and dislocation strain (DS). ${ }^{15}$ Relaxation time of dislocation scattering can be considered as

Dislocation core phonon scattering

$$
\tau_{D C}^{-1}=N_{D} \frac{\bar{V}^{4 / 3}}{v^{2}} \omega^{3} \text {, and }
$$


dislocation strain phonon scattering

$$
\tau_{D S}^{-1}=0.6 B_{D}^{2} N_{D}(\gamma+\Delta \gamma)^{2} \omega\left\{\frac{1}{2}+\frac{1}{24}\left(\frac{1-2 r}{1-r}\right)^{2}\left[1+\sqrt{2}\left(\frac{v_{\square}}{v_{\perp}}\right)\right]^{2}\right\},
$$

where $N_{D}$ is the dislocation density, $B_{D}$ is the effective Burger's vector, $r$ is the Poisson's ratio and $\Delta \gamma$ is the change in $\gamma$ due to the dislocation strain, as given by

$$
\Delta \gamma=\frac{V_{B T} c_{0} K}{k_{B} T_{a}}\left(\gamma \alpha^{2}-\alpha \beta\right)
$$

with

$$
\begin{gathered}
\alpha=\frac{V_{B S}-V_{B T}}{V_{B T}}, \text { and } \\
\beta=\frac{M_{B T}-M_{B S}}{2 M_{B T}},
\end{gathered}
$$

where $c_{0}$ is the concentration of $\mathrm{Bi}_{2} \mathrm{Se}_{3}$ in $\mathrm{Bi}_{2} \mathrm{Te}_{3-\mathrm{x}} \mathrm{Se}_{\mathrm{x}}, K$ is the bulk modulus of $\mathrm{Bi}_{2} \mathrm{Te}_{3}, T_{a}$ is the sample sintering temperature, $V_{B S}$ and $V_{B T}$ are the atomic volume of $\mathrm{Bi}_{2} \mathrm{Se}_{3}$ and $\mathrm{Bi}_{2} \mathrm{Te}_{3}$, and $M_{B S}$ and $M_{B T}$ are the atomic mass of $\mathrm{Bi}_{2} \mathrm{Se}_{3}$ and $\mathrm{Bi}_{2} \mathrm{Te}_{3}$.

According to the Matthiessen rule, ${ }^{15}$ the total scattering relaxation time $\tau_{\text {tot }}$ can be given by $\mathrm{U}+\mathrm{E}+\mathrm{PD}+\mathrm{B}$ mode

$$
\tau_{t o t}^{-1}=\tau_{U}^{-1}+\tau_{E}^{-1}+\tau_{P D}^{-1}+\tau_{B}^{-1}, \text { and }
$$

$\mathrm{U}+\mathrm{E}+\mathrm{PD}+\mathrm{B}+\mathrm{D}$ model 


$$
\tau_{\text {tot }}^{-1}=\tau_{U}^{-1}+\tau_{E}^{-1}+\tau_{P D}^{-1}+\tau_{B}^{-1}+\tau_{D C}^{-1}+\tau_{D S}^{-1} .
$$

By inputting physical parameters listed in Table $\mathrm{S} 2$ into above equations, $\kappa_{l}$ can be calculated by models of $\mathrm{U}+\mathrm{E}+\mathrm{PD}+\mathrm{B}$ and $\mathrm{U}+\mathrm{E}+\mathrm{PD}+\mathrm{B}+\mathrm{D}$. 
Table S2 Physical properties used to calculate $\kappa_{l}$ based on various phonon scattering processes.

\begin{tabular}{|c|c|}
\hline Parameters & Values \\
\hline Debye temperature $\theta_{D}(\mathrm{~K})$ & $164^{16}$ \\
\hline Longitudinal sound velocity $v_{L}\left(\mathrm{~ms}^{-1}\right)$ & $2800^{16}$ \\
\hline Transverse sound velocity $v_{T}\left(\mathrm{~ms}^{-1}\right)$ & $1600^{16}$ \\
\hline Sound velocity $v\left(\mathrm{~ms}^{-1}\right)$ & 1778 \\
\hline Average atomic mass of $\mathrm{Bi}_{2} \mathrm{Te}_{3} M_{B T}(\mathrm{~kg})$ & $2.66 \times 10^{-25}$ \\
\hline Average atomic mass of $\mathrm{Bi}_{2} \mathrm{Se}_{3} M_{B S}(\mathrm{~kg})$ & $2.18 \times 10^{-25}$ \\
\hline Average atomic volume of $\mathrm{Bi}_{2} \mathrm{Te}_{3} V_{B T}\left(\mathrm{~m}^{3}\right)$ & $3.48 \times 10^{-29}$ \\
\hline Average atomic volume of $\mathrm{Bi}_{2} \mathrm{Se}_{3} V_{B S}\left(\mathrm{~m}^{3}\right)$ & $3.19 \times 10^{-29}$ \\
\hline Sample density $\rho$ of $\mathrm{Bi}_{2} \mathrm{Te}_{3}\left(\mathrm{~g} \mathrm{~cm}^{-3}\right)$ & 7.6 \\
\hline Sample density $\rho$ of $\mathrm{Bi}_{2} \mathrm{Te}_{2.7} \mathrm{Se}_{0.3}\left(\mathrm{~g} \mathrm{~cm}^{-3}\right)$ & 7.1 \\
\hline Grain size $d$ for $\mathrm{Bi}_{2} \mathrm{Te}_{2.7} \mathrm{Se}_{0.3}(\mathrm{~nm})$ & $800(\operatorname{Exp})$ \\
\hline Point defect scattering parameter $\Gamma$ & 0.18 (fitted) \\
\hline Dislocation density $N_{D}$ of $\mathrm{Bi}_{2} \mathrm{Te}_{2.7} \mathrm{Se}_{0.3}\left(\mathrm{~cm}^{-2}\right)$ & $1.7 \times 10^{11}(\operatorname{Exp})$ \\
\hline Magnititude of Burger's vector $B_{D}$ of $\mathrm{Bi}_{2} \mathrm{Te}_{2.7} \mathrm{Se}_{0.3}(\AA)$ & 12 (fitted) \\
\hline Poisson's ratio $r$ & $0.4^{17}$ \\
\hline Grüneisen parameter $\gamma$ & $1.5^{18}$ \\
\hline Bulk modulus $K(\mathrm{GPa})$ & $37.4^{16}$ \\
\hline
\end{tabular}


Table S3 Grain size $(d)$ and point defect scattering parameter $(\Gamma)$ for the reported ingot, reported ball milling and our nanostrucutred $\mathrm{Bi}_{2} \mathrm{Te}_{2.7} \mathrm{Se}_{0.3}$.

\begin{tabular}{cccc}
\hline & Ingot $^{4}$ & Ball milling $^{19}$ & Nanostructures \\
\hline$d(\mu \mathrm{m})$ & 75 (fitted) & 10 (fitted) & 0.8 (Exp) \\
$\Gamma$ & 0.07 (fitted) & 0.12 (fitted) & 0.18 (fitted) \\
\hline
\end{tabular}




\section{Hall Coefficient $\left(\boldsymbol{R}_{H}\right)$}

To determined Hall carrier concentration $\left(n_{H}\right)$ and Hall carrier mobility $\left(\mu_{H}\right)$, we measured Hall coefficient $R_{H}$ using the Van der Pauw method in a magnetic field up to $\pm 2 \mathrm{~T} .{ }^{20}$ Figure $\mathrm{S} 14$ plots the measured results, in which the negative sign of measured $R_{H}$ suggests the $n$-type feature for our pellets, which is consistent with the conclusion made from measured $S$. For simplicity, we use the absolute value of measured $R_{H}$ to calculated $n_{H}$ and $\mu_{H}$ by $^{20}$

$$
\begin{gathered}
n_{H}=\frac{1}{e\left|R_{H}\right|}, \text { and } \\
\mu_{H}=\sigma\left|R_{H}\right| .
\end{gathered}
$$

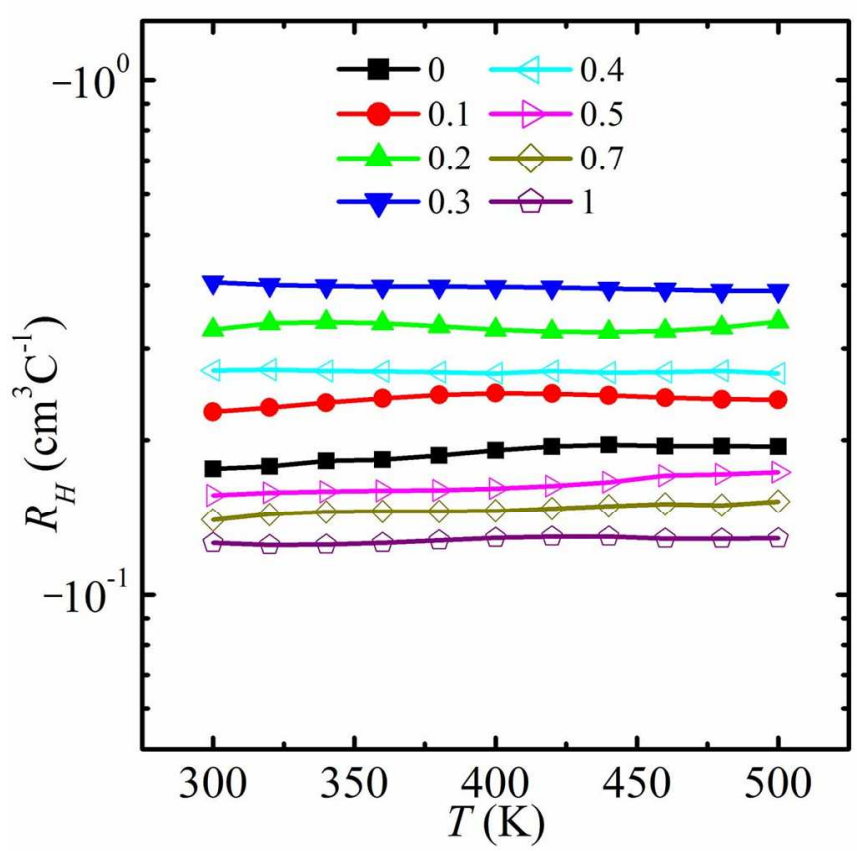

Figure S14 Measured Hall coefficient $\left(R_{H}\right)$ for sintered $\mathrm{Bi}_{2} \mathrm{Te}_{3-\mathrm{x}} \mathrm{Se}_{\mathrm{x}}$ pellets as a function of temperature. 

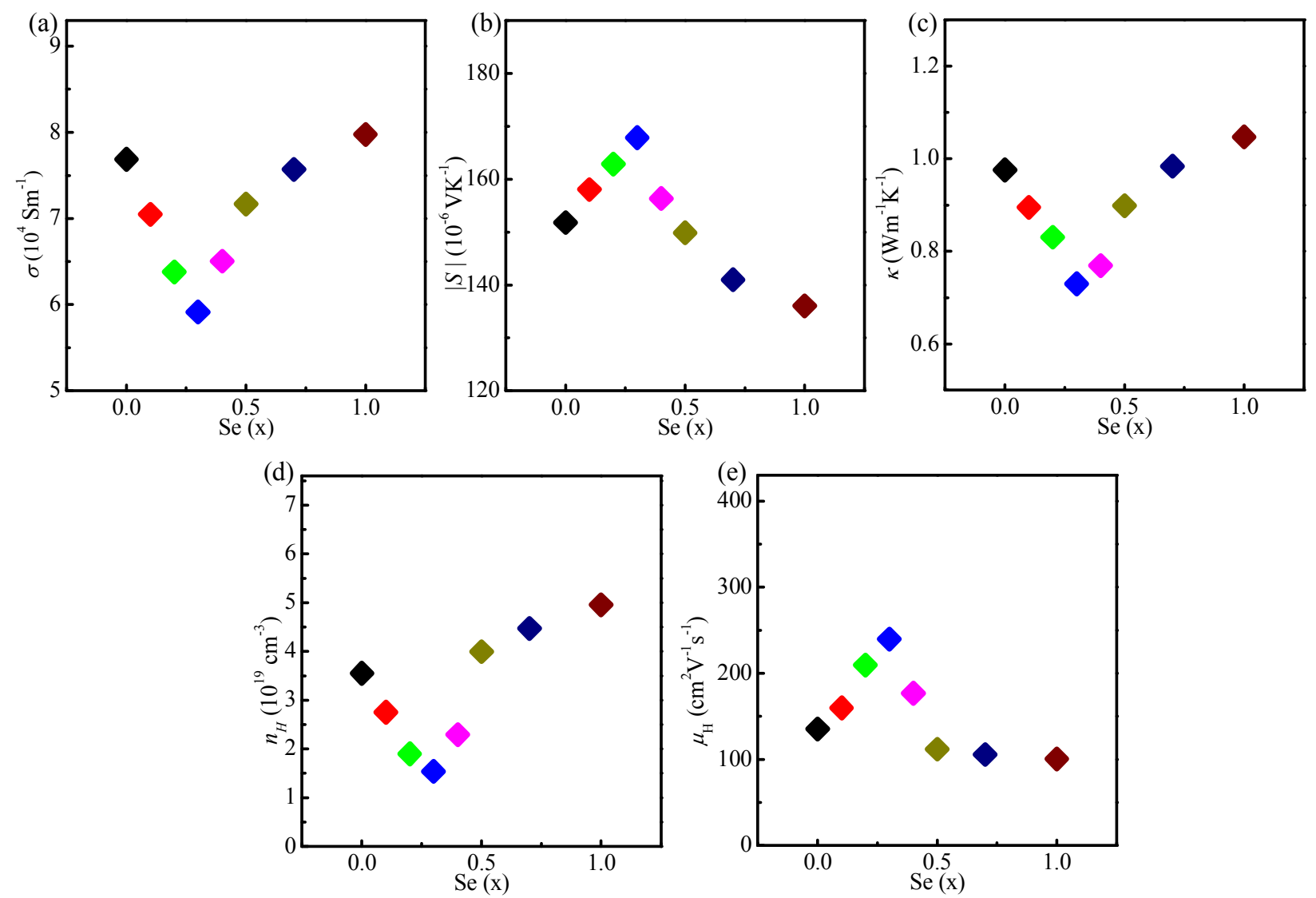

Figure S15 Se content dependent (a) $\sigma$, (b) $S$, (c) $\kappa$, (d) $n_{H}$, and (e) $\mu_{H} \kappa$ for our $\mathrm{Bi}_{2} \mathrm{Te}_{3-\mathrm{x}} \mathrm{Se}_{\mathrm{x}}$ nanostructures at $300 \mathrm{~K}$. 

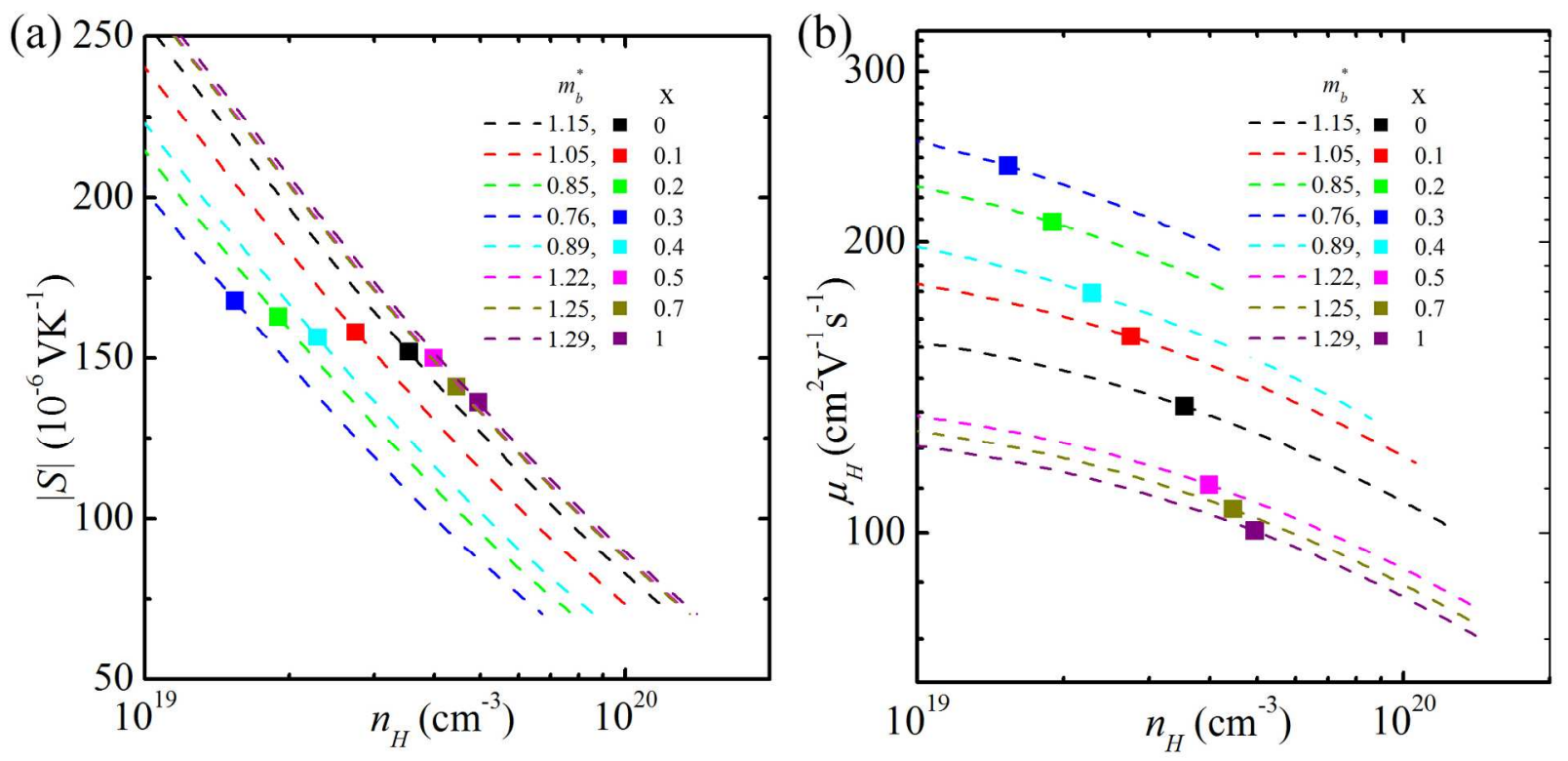

Figure S16 (a) Data points of $|S|$ versus $n_{H}$ compared with the theoratical curves of $|S|$ as a function of $n_{H}$ calculated with the corresponding $m_{b}^{*}$ using single parabolic band model. (b) Data points of $\mu_{H}$ versus $n_{H}$ compared with the theoretical curves of $\mu_{H}$ as a function of $n_{H}$ calculated with the corresponding $m_{b}^{*}$ using single parabolic band model. 


\section{Determination of the Carrier Scattering Mechanism and Energy Gaps}

To determine the carrier scattering types and energy band gaps, we measured the optical properties of the $\mathrm{Bi}_{2} \mathrm{Te}_{3-\mathrm{x}} \mathrm{Se}_{\mathrm{x}}$ nanoplates. Figure $\mathrm{S} 17$ shows the normalized absorption spectra of the $\mathrm{Bi}_{2} \mathrm{Te}_{3-\mathrm{x}} \mathrm{Se}_{\mathrm{x}}$ nanoplates within in a small region $(0.1-0.5 \mathrm{eV})$ of the phonon energy $(\hbar \omega)$. For each sample, the onset of the interband absorption was found in the range of $0.2-0.3 \mathrm{eV}$.

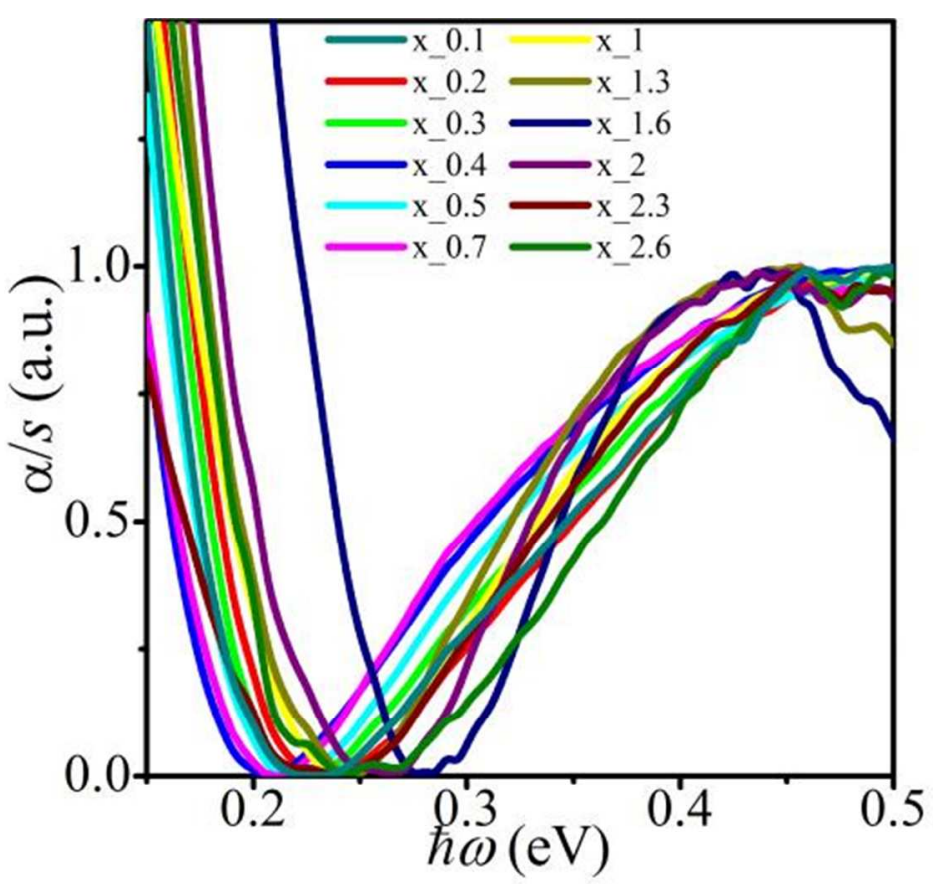

Figure S17 Normalized absorption spectra of $\mathrm{Bi}_{2} \mathrm{Te}_{3-x} \mathrm{Se}_{\mathrm{x}}$ nanoplates.

To determine the carrier scattering types, we need to examine the free carrier (FC) absorption tails. Figure S18 shows the absorption spectrum measured from the nanoplates with the composition of $x=1$. As can be seen, the two different regimes: the intraband regime and the interband regime, separated by an absorption minimum at $\sim 0.2 \mathrm{eV}$. It has been well documented that, for metals and crystalline semiconductors, the reduction of the FC absorption coefficient $(\alpha)$ with frequency $(\omega)$ (i.e. the curve between the FC absorption peak and the separation between 
intraband and interband) follows a relationship of $\alpha \propto\left(\omega \tau_{0}\right)^{-r}$, where $\tau_{0}$ is the average FC relaxation time, and $r$ is the scattering exponent. ${ }^{21}$ In fact, the value of $r$ reflects the scattering types. Applying quantum mechanics, $r=1.5$ when the acoustic phonon scattering dominates, $r=$ 2.5 when the optical phonon scattering dominates, or $r=3$ (3.5) when the ionized impurity scattering dominates. ${ }^{22-24}$ In practice, all these processes may exist. ${ }^{25}$ Using simple power law to fit the corresponding FC absorption tails $\left(\alpha_{F C}\right)$ versus photon energy $(\hbar \omega)$ in the form of $\alpha_{F C}=\alpha_{0}+C(\hbar \omega)^{-r}$ (where $\alpha_{0}$ represents background correction term, and $C$ represents the fitting coefficient), one may extract $r$ values and the information about the scattering process for our nanoplates.

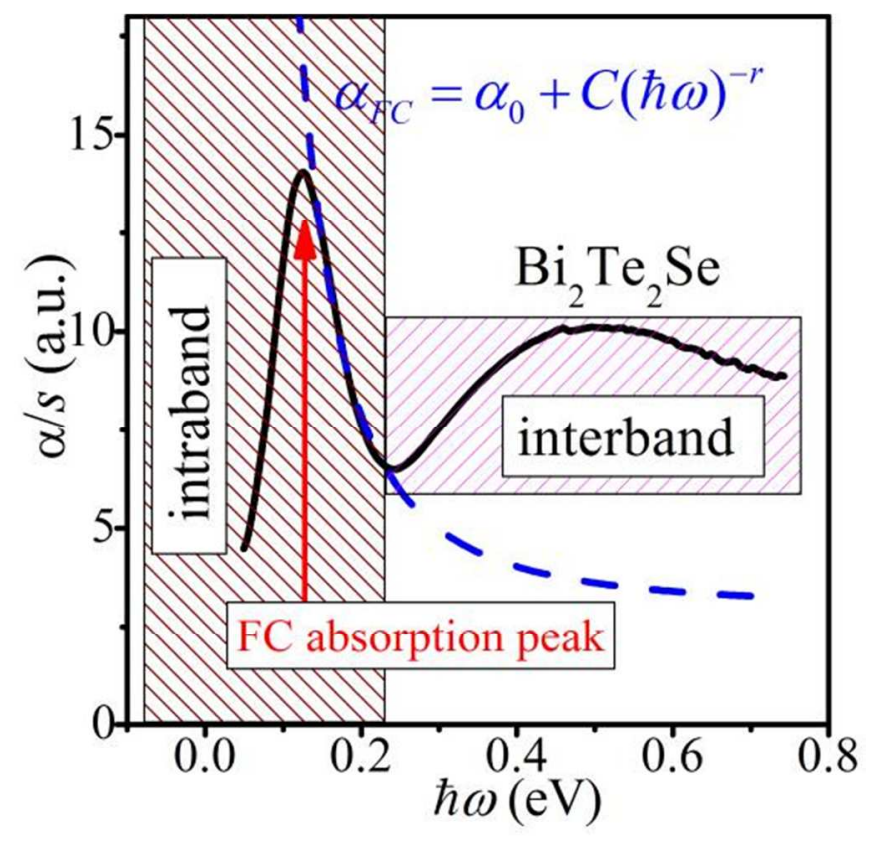

Figure S18 Raw absorption data collected from the $\mathrm{Bi}_{2} \mathrm{Te}_{2} \mathrm{Se}$ nanoplates with the dashed line showing the best fit power law to the FC absorption tail. 
The optical band gaps $\left(E_{g, \text { opt }}\right)$ for our $\mathrm{Bi}_{2} \mathrm{Te}_{3-\mathrm{x}} \mathrm{Se}_{\mathrm{x}}$ nanoplates can be obtained from the absorption spectra of Figure $\mathrm{S} 17$. Since $\mathrm{Bi}_{2} \mathrm{Te}_{3}$ is an indirect semiconductor while $\mathrm{Bi}_{2} \mathrm{Se}_{3}$ is a direct one, ${ }^{26}$ with the direct-indirect transition taking place at $\mathrm{Bi}_{2} \mathrm{Te}_{2.7} \mathrm{Se}_{0.3},{ }^{27}$ we can determine $E_{g, o p t}$ for different $\mathrm{Bi}_{2} \mathrm{Te}_{3-\mathrm{x}} \mathrm{Se}_{\mathrm{x}}$ nanoplates using different models. In the case of indirect band gap $(\mathrm{x}<0.3)$, values of $E_{g, o p t}$ can be determined by extrapolating the linear part of the $\alpha^{1 / 2}$ against $\hbar \omega$ to zero (refer to Figure S19a as an example with $x=0.1)$, whereas in the case of direct band gap $(x \geq 0.3)$, $E_{\mathrm{g}, o p t}$ can be obtained from fitting of the $\alpha^{2}$ against $\hbar \omega$ (refer to Figure $\mathrm{S} 19 \mathrm{~b}$ as an example with $\mathrm{x}$ $=0.3){ }^{28}$
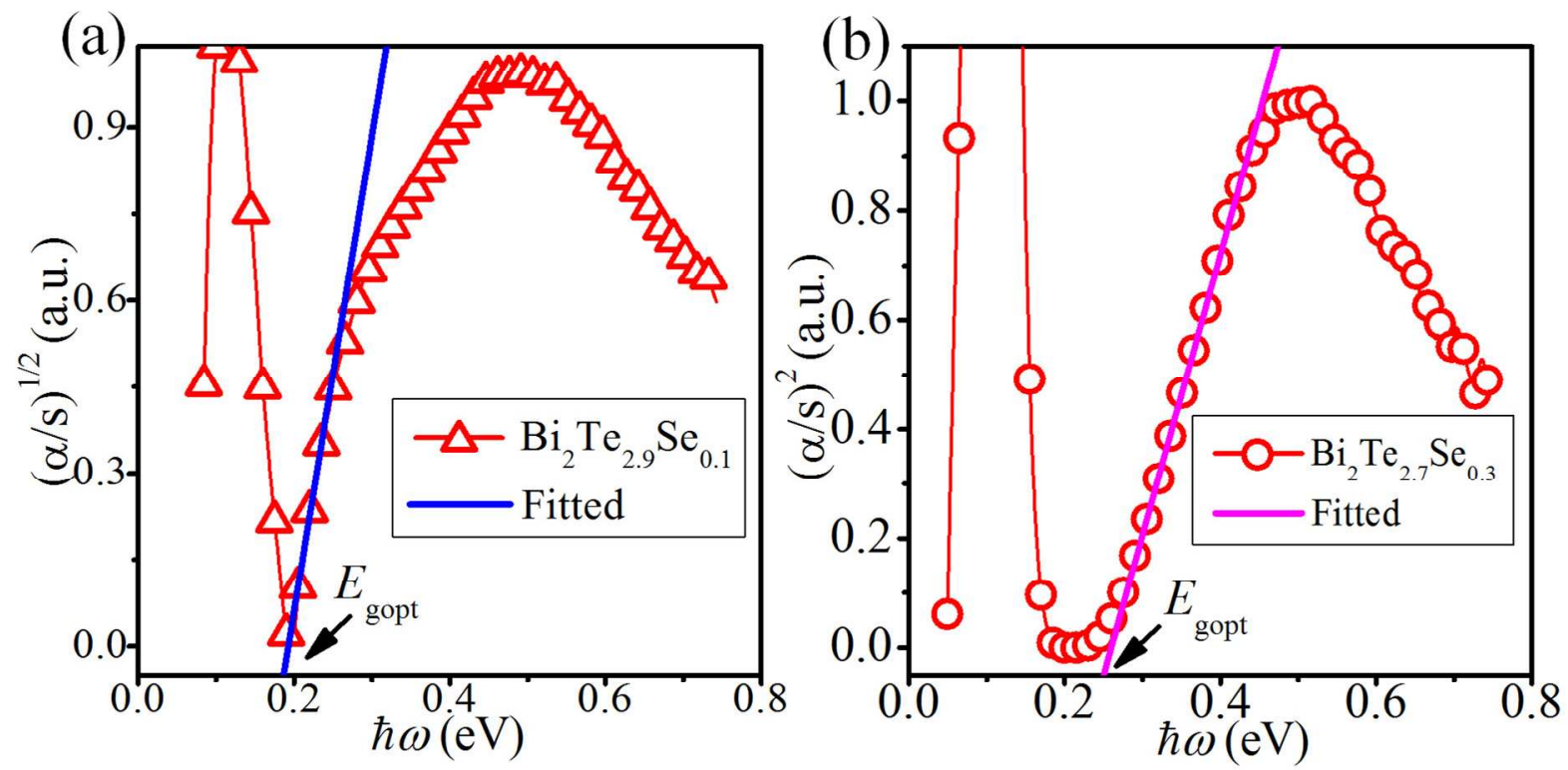

Figure S19 The absorption spectrum for (a) the $\mathrm{Bi}_{2} \mathrm{Te}_{2.7} \mathrm{Se}_{0.1}$ nanoplates using the indirect gap model, and (b) the $\mathrm{Bi}_{2} \mathrm{Te}_{2.9} \mathrm{Se}_{0.3}$ nanoplates using the direct gap model. 


\section{REFERENCES}

(1) Soni, A.; Zhao, Y. Y.; Yu, L. G.; Aik, M. K. K.; Dresselhaus, M. S.; Xiong, Q. H. Enhanced Thermoelectric Properties of Solution Grown $\mathrm{Bi}_{2} \mathrm{Te}_{3-\mathrm{x}} \mathrm{Se}_{\mathrm{x}}$ Nanoplatelet Composites. Nano Lett. 2012, 12, 1203-1209.

(2) Lotgering, F. K. Topotactical Reactions with Ferrimagnetic Oxides Having Hexagonal Crystal Structures-I. J. Inorg. Nucl. Chem. 1959, 9, 113-123.

(3) Prokofieva, L. V.; Pshenay-Severin, D. A.; Konstantinov, P. P.; Shabaldin, A. A. Optimum Composition of a $\mathrm{Bi}_{2} \mathrm{Te}_{3-\mathrm{x}} \mathrm{Se}_{\mathrm{x}}$ Alloy for the $n$-Type Leg of a Thermoelectric Generator. Semiconductors 2009, 43, 973-976.

(4) Wang, S.; Tan, G.; Xie, W.; Zheng, G.; Li, H.; Yang, J.; Tang, X. Enhanced Thermoelectric Properties of $\mathrm{Bi}_{2}\left(\mathrm{Te}_{1-\mathrm{x}} \mathrm{Se}_{\mathrm{x}}\right)_{3}$-Based Compounds as $n$-Type Legs for Low-Temperature Power Generation. J. Mater. Chem. 2012, 22, 20943-20951.

(5) Yan, X.; Poudel, B.; Ma, Y.; Liu, W. S.; Joshi, G.; Wang, H.; Lan, Y.; Wang, D.; Chen, G.;

Ren, Z. F. Experimental Studies on Anisotropic Thermoelectric Properties and Structures of $n$ Type $\mathrm{Bi}_{2} \mathrm{Te}_{2.7} \mathrm{Se}_{0.3}$. Nano Lett. 2010, 10, 3373-3378.

(6) Hu, L.; Wu, H.; Zhu, T.; Fu, C.; He, J.; Ying, P.; Zhao, X. Tuning Multiscale Microstructures to Enhance Thermoelectric Performance of $n$-Type Bismuth-Telluride-Based Solid Solutions. Adv. Energy Mater. 2015, 5, 1500411.

(7) Liu, W.-S.; Zhang, Q.; Lan, Y.; Chen, S.; Yan, X.; Zhang, Q.; Wang, H.; Wang, D.; Chen, G.; Ren, Z. Thermoelectric Property Studies on Cu-Doped n-Type $\mathrm{Cu}_{\mathrm{x}} \mathrm{Bi}_{2} \mathrm{Te}_{2.7} \mathrm{Se}_{0.3}$ Nanocomposites. Adv. Energy Mater. 2011, 1, 577-587. 
(8) Zhao, L.; Lo, S. H.; He, J.; Li, H.; Biswas, K.; Androulakis, J.; Wu, C.-I.; Hogan, T. P.; Chung, D.-Y.; Dravid, V. P.; Kanatzidis, M. G. High Performance Thermoelectrics from EarthAbundant Materials: Enhanced Figure of Merit in PbS by Second Phase Nanostructures. J. Am. Chem. Soc. 2011, 133, $20476-20487$.

(9) Chen, C. L.; Wang, H.; Chen, Y. Y.; Day, T.; Snyder, G. J. Thermoelectric Properties of $p$ Type Polycrystalline SnSe Doped with Ag. J. Mater. Chem. A 2014, 2, 11171-11176.

(10) Hong, M.; Chen, Z.-G.; Yang, L.; Han, G.; Zou, J. Enhanced Thermoelectric Performance of Ultrathin $\mathrm{Bi}_{2} \mathrm{Se}_{3}$ Nanosheets through Thickness Control. Adv. Electron. Mater. 2015, 1, 1500025.

(11) Callaway, J.; von Baeyer, H. Effect of Point Imperfections on Lattice Thermal Conductivity. Phys. Rev. 1960, 120, 1149-1154.

(12) Bessas, D.; Sergueev, I.; Wille, H. C.; Perßon, J.; Ebling, D.; Hermann, R. P. Lattice Dynamics in $\mathrm{Bi}_{2} \mathrm{Te}_{3}$ and $\mathrm{Sb}_{2} \mathrm{Te}_{3}$ : Te and $\mathrm{Sb}$ Density of Phonon States. Phys. Rev. B 2012, 86, 224301.

(13) Zhu, T. J.; Fu, C. G.; Xie, H. H.; Liu, Y. T.; Feng, B.; Xie, J.; Zhao, X. B. Lattice Thermal Conductivity and Spectral Phonon Scattering in FeVSb-Based Half-Heusler Compounds. EPL 2013, 104, 46003.

(14) Xie, H.; Wang, H.; Pei, Y.; Fu, C.; Liu, X.; Snyder, G. J.; Zhao, X.; Zhu, T. Beneficial Contribution of Alloy Disorder to Electron and Phonon Transport in Half-Heusler Thermoelectric Materials. Adv. Funct. Mater. 2013, 23, 5123-5130.

(15) Kim, S. I.; Lee, K. H.; Mun, H. A.; Kim, H. S.; Hwang, S. W.; Roh, J. W.; Yang, D. J.; Shin, W. H.; Li, X. S.; Lee, Y. H.; Snyder, G. J.; Kim, S. W. Dense Dislocation Arrays 
Embedded in Grain Boundaries for High-Performance Bulk Thermoelectrics. Science 2015, 348, $109-114$.

(16) Jenkins, J.; Rayne, J.; Ure, R. Elastic Moduli and Phonon Properties of $\mathrm{Bi}_{2} \mathrm{Te}_{3}$. Phys. Rev. $B$ 1972, 5, 3171-3184.

(17) Clin, T.; Turenne, S.; Vasilevskiy, D.; Masut, R. A. Numerical Simulation of the Thermomechanical Behavior of Extruded Bismuth Telluride Alloy Module. J. Electron. Mater. 2009, 38, 994-1001.

(18) Chen, X.; Zhou, H. D.; Kiswandhi, A.; Miotkowski, I.; Chen, Y. P.; Sharma, P. A.; Lima Sharma, A. L.; Hekmaty, M. A.; Smirnov, D.; Jiang, Z. Thermal Expansion Coefficients of $\mathrm{Bi}_{2} \mathrm{Se}_{3}$ and $\mathrm{Sb}_{2} \mathrm{Te}_{3}$ Crystals from $10 \mathrm{~K}$ to $270 \mathrm{~K}$. Appl. Phys. Lett. 2011, 99, 261912.

(19) Liu, W.; Lukas, K. C.; McEnaney, K.; Lee, S.; Zhang, Q.; Opeil, C. P.; Chen, G.; Ren, Z. Studies on the $\mathrm{Bi}_{2} \mathrm{Te}_{3}-\mathrm{Bi}_{2} \mathrm{Se}_{3}-\mathrm{Bi}_{2} \mathrm{~S}_{3}$ System for Mid-Temperature Thermoelectric Energy Conversion. Energy Environ. Sci. 2013, 6, 552-560.

(20) Borup, K. A.; Toberer, E. S.; Zoltan, L. D.; Nakatsukasa, G.; Errico, M.; Fleurial, J.-P.; Iversen, B. B.; Snyder, G. J. Measurement of the Electrical Resistivity and Hall Coefficient at High Temperatures. Rev. Sci. Instrum. 2012, 83, 123902.

(21) Shimakawa, K.; Itoh, T. Grain Boundary Scattering of Free Electrons in Ga-Doped Microcrystalline Zinc Oxide Films. Jpn. Appl. Phys. 2007, 46, L577-L579.

(22) Walukiewicz, W.; Lagowski, L.; Jastrzebski, L.; Lichtensteiger, M.; Gatos, H. C. Electron Mobility and Free Carrier Absorption in GaAs - Determination of the Compensation Ratio. $J$. Appl. Phys. 1979, 50, 899-908.

(23) Pankove, J. I. Optical Processes in Semiconductors. Dover: New York, 1971.

(24) Fan, H. Y. Infra-Red Absorption in Semiconductors. Rep. Prog. Phys. 1956, 19, 107. 
(25) Osamura, K.; Murakami, Y. Free Carrier Absorption in n-GaAs. Jpn. J. Appl. Phys. 1972, $11,365-371$.

(26) Greenaway, D. L.; Harbeke, G. Band Structure of Bismuth Telluride, Bismuth Selenide and Their Respective Alloys. J. Phys. Chem. Solids 1965, 26, 1585-1604.

(27) Veis, A. N.; Luk’yanova, L. N.; Kutasov, V. A. Band Gap and Type of Optical Transitions at the Interband Absorption Edge in Solid Solutions Based on Bismuth Telluride. Phys. Solid State 2012, 54, 2182-2188.

(28) Scanlon, W. W. Recent Advances in the Optical and Electronic Properties of PbS, PbSe, PbTe and Their Alloys. J. Phys. Chem. Solids 1959, 8, 423-428. 